Supplement of Geosci. Model Dev., 9, 2459-2470, 2016

http://www.geosci-model-dev.net/9/2459/2016/

doi:10.5194/gmd-9-2459-2016-supplement

(c) Author(s) 2016. CC Attribution 3.0 License.

(c) (i)

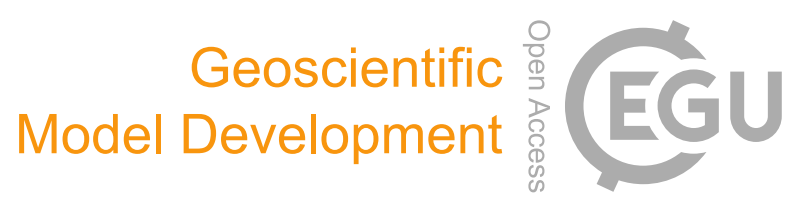

Supplement of

\title{
A consistent prescription of stratospheric aerosol for both radiation and chemistry in the Community Earth System Model (CESM1)
}

\author{
Ryan Reynolds Neely III et al. \\ Correspondence to: R. R. Neely III (r.neely@leeds.ac.uk)
}

The copyright of individual parts of the supplement might differ from the CC-BY 3.0 licence. 
S1. Mie Calculations for Optical Look up Table Code used in CESM1(CAM4)

function mielookupgenerationcam4simplebands

\%Written using Matlab 2014b and utilizing the \% Mie code of Matzler [2002]

load(' volcsulfrefind75-25.mat'); \%Load volcanic aerosol refractive indices from the GEISA spectroscopic database

$\mathrm{r}=.1 \mathrm{E}-9: .1 \mathrm{E}-9: 2000 \mathrm{E}-9 ; \%$ Specify the range of radii

lambda(1,:: $=[200 E-9,245 E-9,265 E-9,275 E-9,285 E-9,295 E-9,305 E-9,350 E-9,640 E-9, \ldots$

2630E-9,4160E-9,4160E-9]; Specify the wavenumber of each band's upper limit

lambda(3,:) $=[245 E-9,265 E-9,275 E-9,285 E-9,295 E-9,305 E-9,350 E-9,640 E-9,700 E-9, \ldots$

2860E-9,4550E-9,4550E-9]; ]; Specify the wavenumber of each band's lower limit

lambda(2,:) $=(\operatorname{lambda}(1,:)+\operatorname{lambda}(3,:)) \cdot / 2$; Calculate the midpoint each band;

massfactor=3./(4.*1.7.*1E3. ${ }^{*}$ ); \%Mass conversion factor for each radii

for range $=1: 3: \%$ For loop for the upper mid and lower wavelengths.

for I=1:length(lambda); \%For loop for each band within the upper lower and mid point ranges.

$\mathrm{x}=\left(2^{*}\right.$ pi./(squeeze $\left(\right.$ lambda(range,I)))). ${ }^{*} \mathrm{r}$; Calculate the size parameter.

Realindex=feval(realindfittedmodel,lambda(range,I)./1E-6); Convert the real index of refraction to correct units

Imagindex=feval(imindfittedmodel,lambda(range,I)./1E-6); Convert the imaginary index of refraction to correct units.

$m=$ complex(Realindex,Imagindex); Use the real and imaginary indices of refraction to create the complex index of refraction

$\%$ Below is copied from Matzler [2002] to use the information above to calculate the Mie parameters

$\mathrm{j}=\mathrm{I}$

for $\mathrm{i}=1$ :length $(\mathrm{x})$

nmax $=\operatorname{round}\left(2+x(i)+4^{*} x(i)^{\wedge}(1 / 3)\right)$

$\mathrm{n} 1=\mathrm{nmax}-1$;

$\mathrm{n}=(1: \mathrm{nmax})$

$\mathrm{cn}=2^{*} \mathrm{n}+1 ; \mathrm{c} 1 \mathrm{n}=\mathrm{n} \cdot{ }^{*}(\mathrm{n}+2) \cdot /(\mathrm{n}+1) ; \mathrm{c} 2 \mathrm{n}=\mathrm{cn} \cdot / \mathrm{n} \cdot /(\mathrm{n}+1)$;

$x 2=x(i)^{*} x(i)$;

$\mathrm{f}=$ Mie_abcd $(\mathrm{m}, \mathrm{x}(\mathrm{i}))$;

anp $=(\operatorname{real}(f(1,:)))$;

anpp $=(\operatorname{imag}(f(1,:)))$

bnp $=(\operatorname{real}(f(2,:)))$

bnpp $=(\operatorname{imag}(f(2,:)))$;

g1 $(1: 4, \mathrm{nmax})=[0 ; 0 ; 0 ; 0] ; \%$ displaced numbers used for

g1 $(1,1: n 1)=a n p(2: n m a x) ; g 1(2,1: n 1)=\operatorname{anpp}(2: n m a x) ; g 1(3,1: n 1)=b n p(2: n m a x) ; g 1(4,1: n 1)=b n p p(2: n m a x)$;

$\mathrm{dn}=\mathrm{cn} .{ }^{*}(\mathrm{anp}+\mathrm{bnp})$;

$q=\operatorname{sum}(d n)$;

qext $=2^{*} \mathrm{q} / \mathrm{x} 2$;

en $=$ cn. ${ }^{*}$ (anp. ${ }^{*} a n p+a n p p .{ }^{*} a n p p+b n p .{ }^{*}$ bnp+bnpp. *bnpp);

$q=\operatorname{sum}(e n)$;

qsca $=2^{*} \mathrm{q} / \mathrm{x} 2$;

qabs=qext-qsca;

$\mathrm{fn}=(\mathrm{f}(1,:)-\mathrm{f}(2,:)) .^{*} \mathrm{cn}$;

$\mathrm{gn}=(-1) \cdot{ }^{\wedge} \mathrm{n}$

$f(3,:)=f n .{ }^{*} g n$;

$q=\operatorname{sum}(f(3,:))$;

$q b=q^{*} q^{\prime} / x 2$;

asy1=c1n. ${ }^{*}\left(\right.$ anp. ${ }^{*} \mathrm{~g} 1(1,:)+$ anpp. ${ }^{*} \mathrm{~g} 1(2,:)+$ bnp. ${ }^{*} \mathrm{~g} 1(3,:)+$ bnpp. $\left.{ }^{*} \mathrm{~g} 1(4,:)\right)$; asy2=c2n. ${ }^{*}\left(\right.$ anp. ${ }^{*}$ bnp+anpp. ${ }^{*}$ bnpp); asy=4/x2 ${ }^{*}$ sum(asy1+asy2)/qsca;

\%Individual calculated parameters are saved to an array.

qratio=qb/qsca;

$\mathrm{Qb}$ (range, $\mathrm{i}, \mathrm{j})=\mathrm{qb}$;

Qext(range,i,j)=qext:

Qabs $($ range,i,j) $=$ qabs

Qsca $($ range $, i, j)=q s c a$;

$X($ range $, i, j)=x(i)$;

$\mathrm{G}($ range, $\mathrm{i}, \mathrm{j})=\mathrm{asy}$

indref(range, $, \mathrm{i}, \mathrm{j})=\mathrm{m}$;

end

clearvars -except Qb r X m Qext Qsca Qabs asy lambda massfactor G range indref realindfittedmodel imindfittedmodel

end

end

w=Qsca./Qext;

$\%$ Array is saved to mat format file.

savefilename=sprintf('/Users/rneely/Dropbox/camRTmieparameters1-9and17-19.mat');

savefile=[savefilename];

save(savefile,'Qb','r','X','indref','Qext','Qsca','Qabs',''G','lambda','w','massfactor') 


\section{S2. Mie Calculations for Optical Look up Table Code CESM1(CAM5)}

function mielookupgenerationrrtmg

\%Written using Matlab 2014b and utilizing the \% Mie code of Matzler [2002]

load(' volcsulfrefind75-25.mat'); \%Load volcanic aerosol refractive indices from the GEISA spectroscopic database

$r=.1 E-9: .1 E-9: 2000 E-9 ; \%$ Specify the range of radii

$\%$ Shortwave Bands

$\%$ comment out next 3 lines calculate long wave parameter file

$\%$ Specify the wavenumber of each bands upper limit

$\operatorname{lambdawn}(1,:)=[2600,3250,4000,4650,5150,6150,7700,8050,12850,16000,22650,29000,38000,820]$;

$\%$ Specify the wavenumber bands lower limit

lambdawn(3,: $=[3250,4000,4650,5150,6150,7700,8050,12850,16000,22650,29000,38000,50000,2600]$

$\%$ Calculate the midpoint of the each band:

lambdawn $(2,:)=(\operatorname{lambdawn}(1,:)+\operatorname{lambdawn}(3,:)) \cdot / 2$;

$\% \mathrm{lw}$

$\%$ Uncomment out next 3 lines calculate long wave parameter file

$\%$ Specify the wavenumber of each bands upper limit

$\%$ lambdawn $(1,:)=[10,350,500,630,700,820,980,1080,1180,1390,1480,1800,2080,2250,2390,2600]$;

$\%$ Specify the wavenumber bands lower limit

$\%$ lambdawn(3,:)=[350, 500, 630, 700, 820, 980, 1080, 1180,1390, 1480, 1800, 2080, 2250, 2390, 2600, 3250];

$\%$ Calculate the midpoint of the each band;

$\%$ lambdawn(2,:) $=($ lambdawn $(1,:)+$ lambdawn $(3,:)) . / 2$

lambda=1e-2./lambdawn; \% Convert wavenumber to wavelength

massfactor=3./(4.*1.75.*1E3. $\left.{ }^{*} \mathrm{r}\right)$;

for range $=1: 3 ; \%$ For loop for the upper mid and lower wavelengths

for I=1:length(lambda); \% For loop for each band within the upper lower and mid point ranges.

$\mathrm{x}=\left(2^{*} \mathrm{pi} . /(\right.$ squeeze $($ lambda $($ range, $\left.\mathrm{I})))\right) .{ }^{*} \mathrm{r}$; Calculate the size parameter.

$\%$ Convert the imaginary index of refraction to correct units.

Realindex=realind(find(realmicron.*1E-6<=lambda(range,I), 1,'first'));

Imagindex=imind(find(immicron. ${ }^{*} 1 \mathrm{E}-6<=$ lambda(range,I),1,'first'));

if isempty (Imagindex $)==1$

Imagindex=4e-6;

end

if isempty $($ Realindex $)==1$

Realindex=1.45;

end

lambda(range,I);

$\%$ Use the real and imaginary indices of refraction to create the complex index of refraction

$\mathrm{m}=$ complex(Realindex, Imagindex)

$\%$ Below is copied from Matzler [2002] to use the information above to calculate the Mie parameters

$j=$;

for $i=1$ :length $(x)$

$n \max =\operatorname{round}\left(2+x(i)+4^{*} x(i)^{\wedge}(1 / 3)\right)$

$\mathrm{n} 1=\mathrm{nmax}-1$;

$\mathrm{n}=(1: \mathrm{nmax})$

$c n=2^{*} n+1 ; c 1 n=n .{ }^{*}(n+2) . /(n+1) ; c 2 n=c n . / n . /(n+1)$;

$x 2=x(i)^{*} x(i)$;

$\mathrm{f}=$ Mie $\operatorname{abcd}(\mathrm{m}, \mathrm{x}(\mathrm{i}))$

anp $=($ real $(f(1,:)))$;

$\operatorname{anpp}=(\operatorname{imag}(f(1,:)))$;

bnp $=(\operatorname{real}(f(2,:)))$;

bnpp $=(\operatorname{imag}(f(2,:)))$

g1 $(1: 4$, nmax $)=[0 ; 0 ; 0 ; 0] ; \%$ displaced numbers used for

g1 $(1,1: n 1)=\operatorname{anp}(2: n m a x) ; g 1(2,1: n 1)=\operatorname{anpp}(2: n m a x) ; g 1(3,1: n 1)=b n p(2: n m a x) ; g 1(4,1: n 1)=b n p p(2: n m a x)$;

$\mathrm{dn}=\mathrm{cn} .{ }^{*}(\mathrm{anp}+\mathrm{bnp})$;

$\mathrm{q}=\operatorname{sum}(\mathrm{dn})$

$\mathrm{qext}=2^{*} \mathrm{q} / \mathrm{x} 2$;

en $=$ cn. ${ }^{*}\left(a n p .{ }^{*} a n p+a n p p .{ }^{*} a n p p+b n p .{ }^{*} b n p+b n p p .{ }^{*} b n p p\right)$;

$\mathrm{q}=\operatorname{sum}(\mathrm{en})$;

qsca $=2^{*} \mathrm{q} / \mathrm{x} 2$;

qabs=qext-qsca;

$\mathrm{fn}=(\mathrm{f}(1,:)-\mathrm{f}(2,:)) .{ }^{*} \mathrm{cn}$;

gn $=(-1) .^{\wedge} \mathrm{n}$;

$f(3,:)=f n . * g n ;$

$\mathrm{q}=\operatorname{sum}(\mathrm{f}(3,:))$

$q b=q^{*} q^{1} / x 2$;

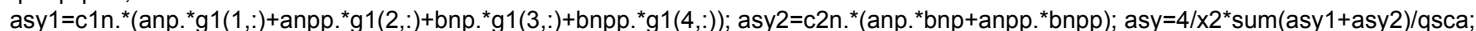

qratio=qb/qsca;

\% Individual calculated parameters are saved to an array.

$\mathrm{Qb}($ range, $\mathrm{i}, \mathrm{j})=\mathrm{qb}$;

Qext(range,i,j)=qext;\%*massfactor(i);

Qabs $($ range,i,j) $=$ qabs; \%*massfactor(i);

Qsca(range,i,j)=qsca;\%*massfactor(i);

$X($ range $, i, j)=x(i)$

$\mathrm{G}($ range,i,j)=asy;

indref(range, $\mathrm{i}, \mathrm{j})=\mathrm{m}$;

end

clearvars -except Qb r X m Qext Qsca Qabs asy lambda massfactor G range indref realmicron immicron realind imind

end

end

w=Qsca./Qext;

$\%$ Array is saved to mat format file.

savefilename=sprintf('/Users/rneely/Dropbox/camRRTMGsw.mat'); \%change sw to Iw to save long wave parameter file

savefile=[savefilename]

save(savefile,'Qb','r','X','indref','Qext','Qsca','Qabs','G','lambda','w','massfactor') 


\section{S3. Impact of the Changes in Forcing Files and Optical Paramters on the Simulated SAOD in CAM4 and CAM5}
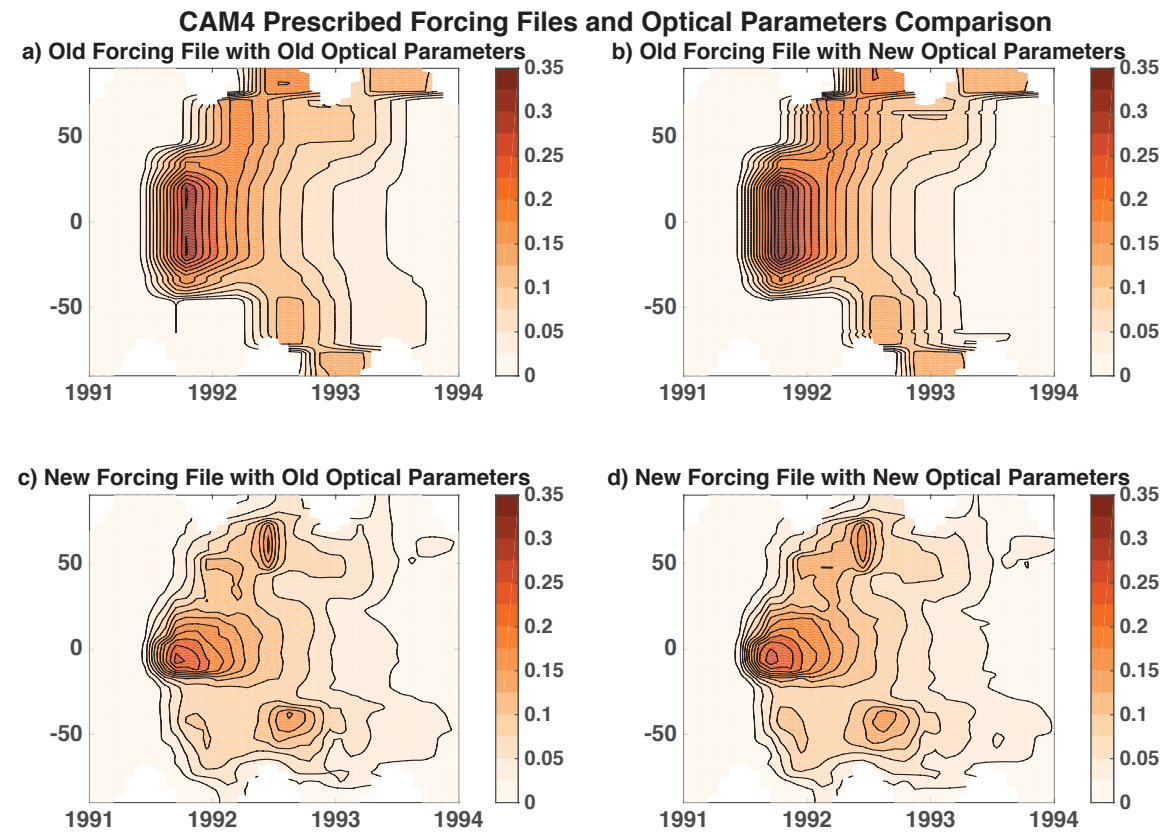

Figure S3.1. Monthly times series comparison of the zonal mean SAOD after the $1991 \mathrm{Mt}$. Pinatubo eruptions simulated by CAM4 using: a) the old (CCSM4) forcing file and old optical parameters, b) the old forcing file with the new optical parameters (assuming a constant size distribution), c) the new forcing file with old optical parameters (assuming a constant size distribution) and d) the new forcing file with new optical parameters. Results for CAM4 also apply for all model configurations of CESM1 that utilise CAMRT (notably CAM4-chem and WACCM4).
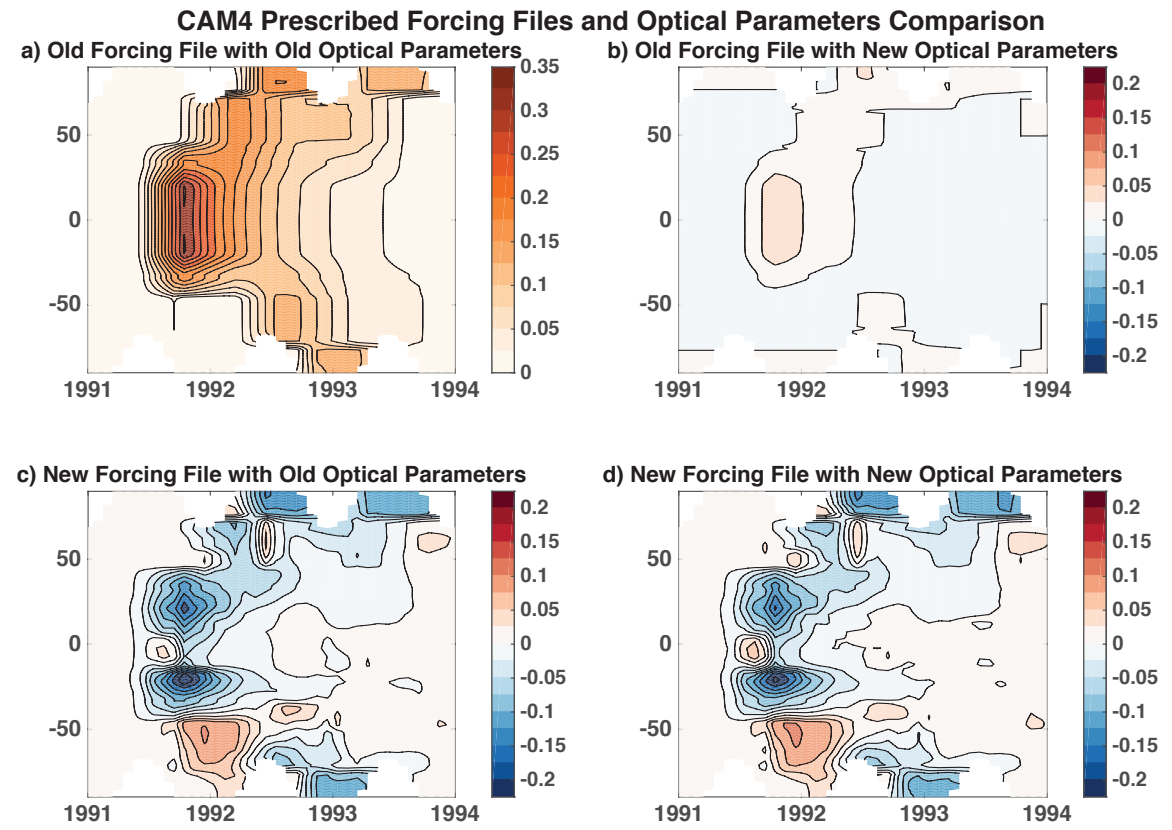

Figure S3.2. Monthly times series of the difference in zonal mean SAOD after the $1991 \mathrm{Mt}$. Pinatubo eruptions simulated by CAM4. Differences are calculated between the original model setup utilising the old forcing file and old optical parameters shown in panel a) with simulations utilising: b) the old forcing file with new optical parameters (assuming a constant size distribution), c) the new forcing file with old optical parameters (assuming a constant size distribution) and d) the new forcing file with new optical parameters. In the differences, a positive value indicates the simulation with changes to the stratospheric aerosol scheme (simulations b, c \& d) has increased SAOD. 

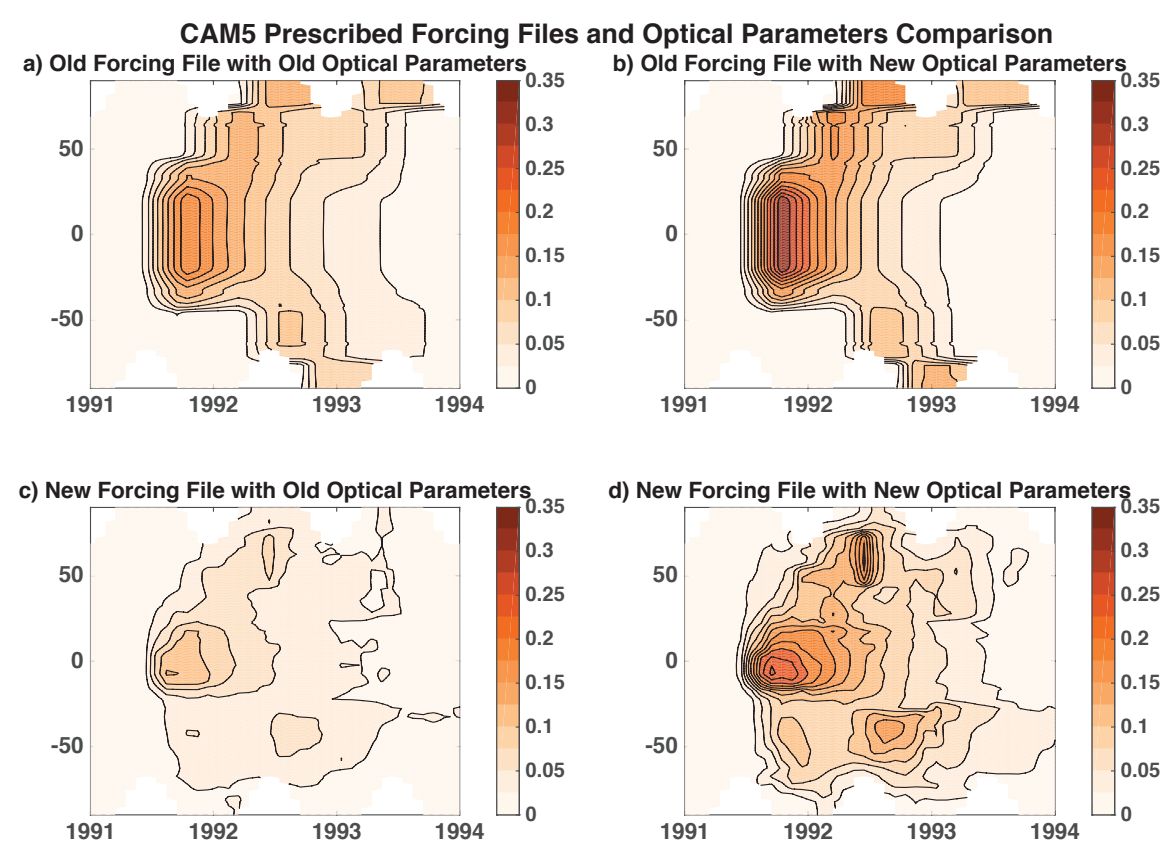

Monthly times series comparison of the zonal mean SAOD after the $1991 \mathrm{Mt}$. Pinatubo eruptions simulated by CAM5 using: a) the old (CCSM4) forcing file and old optical parameters, b) the old forcing file with the new optical parameters (assuming a constant size distribution), c) the new forcing file with old optical parameters (assuming a constant size distribution) and d) the new forcing file with new optical parameters. Results for CAM5 also apply for all model configurations of CESM1 that utilise RRTMGT (notably WACCM5).
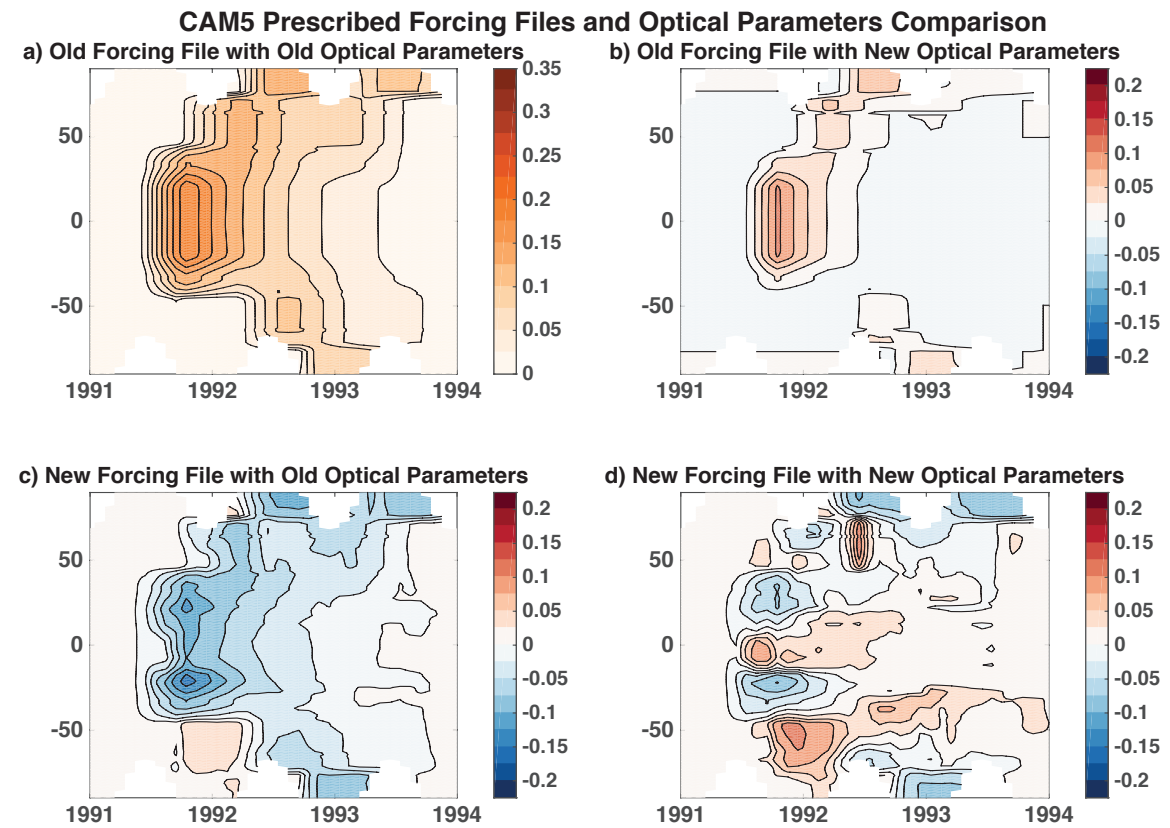

Figure S3.4. Monthly times series of the difference in zonal mean SAOD after the $1991 \mathrm{Mt}$. Pinatubo eruptions simulated by CAM5. Differences are calculated between the original model setup utilising the old forcing file and old optical parameters shown in panel a) with simulations utilising: b) the old forcing file with new optical parameters (assuming a constant size distribution), c) the new forcing file with old optical parameters (assuming a constant size distribution) and d) the new forcing file with new optical parameters. In the differences, a positive value indicates the simulation with changes to the stratospheric aerosol scheme (simulations b, c \& d) has increased SAOD. 
In Figures S3.1 through Figure S3.4, the impact of the change in forcing file and optical parmaters on monthly, zonal mean SAOD is examined for both CAM4 and CAM5 for 3 years following the Mt. Pinatubo. For both CAM4 and CAM5, the new optical parameters, increase the peak SAOD (panel b) compared to panel a) and panel d) compared to panel c) in all Figures). Given the same optical parameters, the new forcing file both changes the peak SOAD after the eruption and subsequent evolution of the SAOD. Evident from these comparisons is the fact that, to first order, having the best estimate of the aerosol mass distribution is most important. 